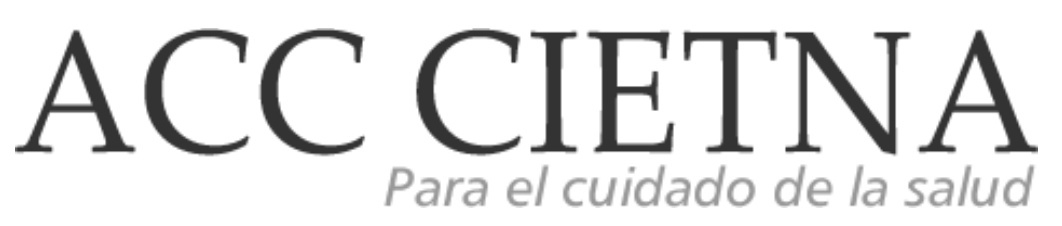

https://doi.org/10.35383/cietna.v8i2.664

e-ISSN: 2309-8570

Universidad Católica Santo Toribio de Mogrovejo

\title{
Aportes de la fenomenología interpretativa a las investigaciones de enfermería y al cuidado enfermero asistencial
}

\author{
Contributions of interpretive phenomenology to nursing research and \\ nursing care
}

\author{
Cinthia Elizabeth González-Soto *, 1, a \\ cinthiag_20@hotmail.com \\ http://orcid.org/0000-0001-9720-5413
}

Diana Laura Molina-Avilez ${ }^{1 \text {, a }}$

lauramolina048@gmail.com

http://orcid.org/0000-0001-7315-5503

Francisco Javier Sabogal Camargo 1, b
fsabogal@javeriana.edu.co
https://orcid.org/0000-0002-1111-0227

Damarys Julissa Baca Guido ${ }^{1, ~ b}$

damarysbaca371@gmail.com

http://orcid.org/0000-0002-7399-8882

\author{
* Autor corresponsal \\ 1 Campus Celaya-Salvatierra, Universidad de \\ Guanajuato, Celaya, Guanajuato, México \\ 2 Pontificia Universidad Javeriana, Bogotá, Colombia \\ a Estudiante de Doctorado en Ciencias de Enfermería \\ ${ }^{b}$ Estudiante de Maestría en Enfermería Oncológica \\ c Estudiante de Maestría en Enfermería del Paciente \\ Crítico
}

\section{Fechas importantes}

Recibido: 2021-06-29

Aceptado: 2021-11-15

Publicado online: 2021-12-16

\begin{abstract}
Resumen
La fenomenología como método de investigación representa una de las herramientas más valiosas para la enfermería pues permite estudiar el principal fenómeno de interés para la disciplina: el cuidado de la experiencia de la salud humana. El presente ensayo tiene por objetivo reflexionar sobre los aportes de la fenomenología interpretativa a las investigaciones de enfermería y al cuidado enfermero asistencial. Se inició con el análisis y la reflexión en base al enfoque de Martin Heidegger respecto al uso de la fenomenología interpretativa como medio de investigación en enfermería. En enfermería, el acto de cuidar implica no solo al paciente sino también a la enfermera como proveedora de cuidado, donde debe reconocer su ser y el del otro para comprender y dar significado a la realidad del paciente, para lograr esta interpretación se precisa del diálogo y la escucha, elementos esenciales en la relación de cuidado enfermera-paciente, estos dos elementos muestran la fuerte relación entre la fenomenología interpretativa y el cuidado de enfermería para construir los significados de los fenómenos del cuidado. Los aportes de la fenomenología interpretativa al cuidado enfermero han sido de gran valor para la mejora del cuidado que se brinda, pues han visibilizado las necesidades más humanas de las personas, aspectos que frecuentemente enfermería ha descuidado.
\end{abstract}

Palabras clave: Fenomenología, fenomenología interpretativa, enfermería 


\begin{abstract}
Phenomenology as a research method represents one of the most valuable tools for nursing as it allows the study of the main phenomenon of interest to the discipline: the care of the human health experience. This paper aims to reflect on the contributions of interpretative phenomenology to nursing research and nursing care. It began with the analysis and reflection based on Martin Heidegger's approach to the use of interpretative phenomenology as a means of nursing research, to continue with the critical analysis of the literature on the topic addressed. In nursing, the act of caring involves not only the patient but also the nurse as a caregiver, where she must recognize her being and that of the other to understand and give meaning to the patient's reality, to achieve this interpretation requires dialogue and listening, essential elements in the nurse-patient care relationship, these two elements show the strong relationship between interpretive phenomenology and nursing care to build the meanings of care phenomena. The contributions of interpretive phenomenology to nursing care have been of great value for the improvement of the care provided, as they have made visible the most human needs of people, aspects that nursing has often neglected.
\end{abstract}

Keywords: Phenomenology, interpretative phenomenology, nursing

\title{
Introducción
}

Desde los inicios de la enfermería como disciplina el interés se centró en el curso del cuidado convirtiéndolo en el deber de ser de la profesión, entendiendo el deber como una voluntad que da dirección ${ }^{1}$. El cuidado para las enfermeras alude a un acto holístico que busca la comprensión del Ser a través de la respuesta a los sufrimientos físicos y emocionales ${ }^{2}$, esta necesidad de comprensión de la experiencia humana ha llevado a la investigación enfermera a explorar metodologías que permitan profundizar en los fenómenos del cuidado y a partir de ello generar conocimiento útil para la práctica enfermera.

En este sentido la fenomenología como método de investigación representa una de las herramientas más valiosas para la enfermería pues permite estudiar el principal fenómeno de interés para la disciplina: el cuidado de la experiencia de la salud humana. A través del método fenomenológico los enfermeros son capaces de incluir esta perspectiva de investigación en cada encuentro con los pacientes, buscando la comprensión de las necesidades de cuidado y aprendiendo de ello para cuidar mejor ${ }^{3}$.

Pese a que la naturaleza de la fenomenología es trascendental, centrada en la esencia que proviene de la descripción, este enfoque fue extendido buscando una naturaleza más ontológica dando origen a la fenomenología interpretativa la cual hace énfasis en la importancia de conectarse con el individuo en numerosos niveles y considerar cuidadosamente la experiencia humana compartida ${ }^{4}$, este enfoque permite a la investigación realizada por enfermería ofrecer una representación creíble y segura de las experiencias de los participantes respaldadas con datos significativos ${ }^{5}$. La fenomenología interpretativa, también conocida como fenomenología hermenéutica, propone una interpretación del mundo que es temporal, es decir, que un fenómeno puede ser visto de diversas maneras dependiendo de cómo se acceda a él o del momento en que sea estudiado ${ }^{6}$.

Gran parte de las investigaciones fenomenológicas realizadas por enfermería se encuentran guiadas por la fenomenología interpretativa, pues permite interpretar la experiencia vivida de las personas cuidadas a través del diálogo que ocurre en los momentos de relación intersubjetiva, donde se brinda el cuidado desde la perspectiva del ser unitario ${ }^{6}$, sin embargo, no todos los abordajes desde la fenomenología son exitosos pues demandan un amplio conocimiento y entrenamiento por parte de los investigadores, por ello, se ha establecido como 
objetivo de este ensayo reflexionar sobre los aportes de la fenomenología interpretativa a las investigaciones de enfermería y al cuidado enfermero asistencial.

\section{Desarrollo}

\section{Fenomenología interpretativa como método de investigación en enfermería}

Parte esencial del cuidado de enfermería debe basarse en la comprensión de las personas, de sus sentimientos y pensamientos ante los diversos procesos de salud y enfermedad que se presentan diariamente. Los aportes teóricos de Heidegger puntualizan en "permitir ver lo que se muestra, tal como se muestra a sí mismo y en cuanto se muestra desde sí mismo", es así como la fenomenología es hermenéutica porque designa el quehacer de la interpretación ${ }^{7}$, por lo tanto, su postura analiza la existencia del ser, al mismo tiempo cuestiona de donde emergen las experiencias. Por esta razón, no sólo se pretende identificar el contexto que define el fenómeno, también concierne a que el investigador comprenda el significado y sea capaz de interpretarlo, alejado de sus ideas a fin de lograr el significado real.

Esta comprensión puede lograrse por medio del estudio de la experiencia vivida respecto a algún fenómeno de cuidado desde la perspectiva del protagonista de la misma a través de la fenomenología, la cual, por su cualidad de profundizar en los fenómenos a partir de los relatos reflexivos de las experiencias vividas se vuelve un método invaluable para generar conocimiento enfermero. Se propone una interpretación temporal del mundo, en la que se modifica de un momento a otro de acuerdo a las características del fenómeno estudiado ${ }^{7} \mathrm{La}$ esencia del ser humano y el método de la fenomenología interpretativa comparten el carácter subjetivo, esto permite a los profesionales de enfermería tener la visión de la persona bajo su cuidado como un ser único, integral y diferente al resto con características y cualidades influidas por su entorno ${ }^{8}$.

Partiendo de esta visión y sumando las habilidades de enfermería para entablar relaciones centradas en el intercambio de experiencias y en la escucha activa de los pacientes, la fenomenología interpretativa como método, parte del paradigma cualitativo en la investigación con bases filosóficas para comprender los fenómenos enfermeros, siendo útil para el desarrollo de enfermería desde una perspectiva inductiva en la práctica disciplinar que responda a las necesidades humanas ${ }^{9,10}$. Este método resulta adecuado para conocer diversos aspectos humanos que en cierta manera fortalecen el objeto de enfermería; el cuidado. En este sentido, la fenomenología interpretativa ha sido una de las más empleadas en estudios fenomenológicos lideradas por profesionales de enfermería en el área clínica o comunitaria ${ }^{9-}$ ${ }^{11}$, resaltando aspectos que ante una enfermedad o riesgo potencial los pacientes tienden a experimentar barreras para llevar a cabo el cuidado de su salud, éstas pueden ser atribuidas a la atención médica o por la propia experiencia de la enfermedad, teniendo un impacto negativo en su bienestar ${ }^{12}$.

Enfatizando en el involucramiento y ejercicio de habilidades que conlleven al cuidado en la diferentes etapas de vida del ser humano, sentando las bases para profundizar en ciertas temáticas y dando origen a valioso conocimiento para mejorar los cuidados enfermeros ${ }^{12,13}$, de ahí la importancia del método interpretativo como directriz para los profesionales en enfermería, especialmente de quienes presentan la inquietud por dedicarse a la investigación científica que provea de habilidad y experiencia a fin de lograr el objetivo de comprender los significados a través de la interpretación. 


\section{Fenomenología interpretativa y cuidado enfermero asistencial}

La fenomenología al ser ampliamente retomada como método de investigación por los enfermeros, ha dado resultados que han revolucionado el cuidado de enfermería centrado en la persona ${ }^{14}$, pese a que el empleo de los métodos cualitativos en investigación de enfermería es relativamente nuevo, cada vez más profesionales se inclinan a retomar nuevas formas de investigar los fenómenos del cuidado ${ }^{10}$, donde como ya se ha mencionado la fenomenología y en especial la fenomenología interpretativa representan una gran herramienta para mejorar el cuidado que brinda enfermería.

Esta fenomenología pone de manifiesto qué hay oculto en la experiencia común diaria, en la estructura de la cotidianidad o ser en el mundo, entendida como un sistema interrelacionado de aptitudes, papeles sociales, proyectos e intenciones ${ }^{15}$. En enfermería, el acto de cuidar implica no solo al paciente sino también a la enfermera como proveedora de cuidado, donde debe reconocer su ser y el del otro para comprender y dar significado a la realidad del paciente, para lograr esta interpretación se precisa del diálogo y la escucha activa, elementos esenciales en la relación de cuidado enfermera-paciente, estos dos elementos muestran la fuerte relación entre la fenomenología interpretativa y el cuidado de enfermería para construir los significados de los fenómenos del cuidado más humanos ${ }^{8}$.

Esta relación aporta un intercambio, el sujeto, vivencias y objeto, el marco del cual existe como ser humano, este enfoque busca redefinir las formas de relación efectiva entre sujeto y objeto que tienen lugar para proclamar los contenidos fenomenológicos. El proceso de cuidar es innato de enfermería y es una de sus actividades principales desde la integración de sus conocimientos, habilidades, intuición y pensamiento crítico; es desde ahí que se construye para mantener y recuperar la experiencia vivida de la persona por medio de la intersubjetividad con las personas que cuida ${ }^{16}$.

La fenomenología interpretativa ha hecho posible la comprensión de variados fenómenos del cuidado enfermero, es posible encontrar publicaciones cuya población de estudio son enfermeros profesionales o estudiantes en enfermería, sin embargo, el área donde se centran este tipo de estudios en la enfermería es sin duda el área asistencial, abordando temáticas tan complejas como la salud mental, el cuidado al final de la vida, la adherencia a los tratamientos, padecimientos estigmatizantes como el VIH, por mencionar algunas. Estas investigaciones son generadoras de herramientas para la mejora del cuidado, ejemplo de ello es una investigación reciente que buscó comprender las experiencias de las personas sobrevivientes de un intento de suicidio y cuyos resultados permitieron mejorar la calidad del cuidado que brinda enfermería, pues se evidenció que estas personas necesitan apoyo, comprensión, empatía y compañía para mitigar el dolor emocional, el cual refieren es mayor que el dolor físico ${ }^{17}$.

Un ejemplo más popular de las bondades de los abordajes de enfermería desde este método es sin duda la investigación de Perez-Toríz ${ }^{18}$ que abordó el cuidado de enfermería durante la pandemia de COVID-19, los resultados de este estudio fenomenológico interpretativo dieron a los profesionales del cuidado herramientas, diferentes a las técnicas y procedimientos médicos, para aliviar el sufrimiento físico y emocional de los pacientes en las áreas de hospitalización.

Con estos breves ejemplos es posible demostrar la importancia de los aportes de la fenomenología interpretativa en el fortalecimiento del cuidado de enfermería, permitiendo a 
los enfermeros comprender y significar la realidad, centrándose en la búsqueda de razones y no la causa de los hechos para alcanzar el conocimiento, siempre fundamentándose en las experiencias vividas y regresando a las cosas mismas para mirarlas tal y como se manifiestan ${ }^{19}$.

De manera similar a lo expuesto, las investigaciones desde la fenomenología interpretativa son más populares en los estudios que los enfermeros realizan en las áreas asistenciales, probablemente debido a la mayor concentración del personal enfermero en centros de salud y hospitales, además que son estas las áreas donde el contacto con las personas es más estrecho y prolongado lo cual propicia la confianza y empatía para lograr profundizar en los fenómenos de cuidado que ahí se manifiestan, sin embargo, este abordaje fenomenológico puede y es retomado por investigaciones enfermeras en otros ámbitos de atención con resultados igualmente exitosos.

\section{Conclusiones}

La reflexión sobre el papel de la fenomenología interpretativa en la investigación de enfermería permite profundizar en los aportes del método al cuidado enfermero, los cuales han sido de gran valor para la mejora del cuidado que se brinda mediante la visibilización de las necesidades más humanas de las personas, aspectos que comúnmente se ignoran o no se cuidan por parte de enfermería, los abordajes bajo el método fenomenológico interpretativo evidencian la necesidad expresada por los pacientes de un cuidado cargado de apoyo, empatía y comprensión.

Este método hace posible el estudio de los fenómenos subjetivos del cuidado, pero también de los fenómenos menos explorados en la relación de cuidado enfermera-paciente, motivo por el cual supone una fuente muy valiosa de conocimientos que permitirán realizar intervenciones en pro de las personas cuidadas, no solo dentro del área asistencial sino en todas las áreas de ejercicio de la enfermería. Analizar estos aspectos evidencia la utilidad que tiene para enfermería el método fenomenológico interpretativo, pues contribuye al conocimiento y comprensión de las experiencias de las personas, es una ventaja importante para contextualizar el cuidado ante las situaciones reales de la práctica e investigación y a la par de ello, brinda oportunidades para ampliar el conocimiento e interés al respecto.

\section{Referencias}

1. Freter B. Nursing as accommodated care: A contribution to the phenomenology of care. Appeal, concern, volition, practice. In: Care in Healthcare: Reflections on Theory and Practice [Internet]. 2017 [Citado 12 Mar 2021]; (1): 37-49. Disponible en: https://doi.org/10.1007/978-3-319-6129113

2. Freysteinson WM. A Synopsis of Ricoeur's Phenomenology of the Will: Implications for Nursing Practice, Research, and Education. J Holist Nurs [Internet]. 2019 [Citado 09 Mar 2021]; 37(1): 8793. Disponible en: https://doi.org/10.1177/0898010118778904

3. Santiago EA, Brown C, Mahmoud R, Carlisle J. Hermeneutic phenomenological human science research method in clinical practice settings: An integrative literature review. Nurse Educ Pract [Internet]. 2020 [Citado 12 Mar 2021]; 47: 102837. Disponible en: https://doi.org/10.1016/j.nepr.2020.102837

4. Burns $M$, Peacock S. Interpretive phenomenological methodologists in nursing: A critical analysis and comparison. Nurs Inq [Internet]. 2019 [Citado 12 Mar 2021]; 26(2): e12280. Disponible en: https://doi.org/10.1111/nin.12280

5. Snelgrove SR. Conducting qualitative longitudinal research using interpretative phenomenological analysis. Nurse Res [Internet]. 2014 [Citado 12 Mar 2021]; 22(1): 20-5. Disponible en: https://doi.org/10.7748/nr.22.1.20.e1277 
6. Ramírez C. Fenomenología hermenéutica y sus implicaciones en enfermería. Index de Enfermería [Internet]. 2016 [Citado 15 Mar 2021]; 25(1-2): 82-5. Disponible en: http://scielo.isciii.es/scielo.php?script=sci_arttext\&pid=S1132-12962016000100019\&lng=es

7. Fuster D. Investigación cualitativa: Método fenomenológico hermenéutico. Propósitos y Representaciones [Internet]. 2019 [Citado 21 Feb 2021]; 7(1): 201-229. Disponible en: http://dx.doi.org/10.20511/pyr2019.v7n1.267

8. Acevedo-Figueroa L, Ávarez-Aguirre A. Vista de Fenomenología: filosofía comprensiva e interpretativa para el cuidado de enfermería. SANUS [Internet]. 2018 [Citado 12 Mar 2021]; 3(7): 68-77. Disponible en: https://sanus.unison.mx/index.php/Sanus/article/view/102/87

9. Tuohy D, Cooney A, Dowling M, Murphy K, Sixsmith J. An overview of interpretive phenomenology as a research methodology. Nurse Res [Internet]. 2013 [Citado 12 Mar 2021]; 20(6): 17-20. Disponible en: https://doi.org/10.7748/nr2013.07.20.6.17.e315

10. Rubio M, Arias M. Fenomenología y conocimiento disciplinar de enfermería. Rev Cubana Enferm [Internet]. 2013 [Citado 07 Abr 2021];29(3). Disponible en: http://scielo.sld.cu/scielo.php?script=sci_arttext\&pid=S0864-03192013000300005

11. Expósito C, Villarreal C, Palmet J, Borja G, Segura B, Sánchez A. La fenomenología, un método para el estudio del cuidado humanizado. Rev Cubana Enferm [Internet]. 2019 [Citado 12 Mar 2021]; 35(1). Disponible en: http://www.revenfermeria.sld.cu/index.php/enf/article/view/2333

12. Nikitara M, Constantinou CS, Andreou E, Latzourakis E, Diomidous M. Views of People with Diabetes Regarding Their Experiences of the Facilitators and Barriers in Type 1 Diabetes Inpatient Care: An Interpretative Phenomenological Analysis. Behav Sci (Basel) [Internet]. 2020 [Citado 07 Abr 2021]; 10(8). Disponible en: https://pubmed.ncbi.nlm.nih.gov/32707985/

13. Pérez $M$, Dias $A D$, Calixto $G$. Experiencia vivida de los adolescentes consumidores de drogas: un abordaje fenomenológico. Rev da Esc Enferm [Internet]. 2018 [Citado 07 Abr 2021]; 52(1): 1-8. Disponible en: http://dx.doi.org/10.1590/S1980-220X2017042803349

14. Albañil-Delgado S, Guerrero-Castañeda R. La entrevista fenomenológica como práctica docente de pregrado en enfermería. Rev Presencia salud Ment Investig y humanidades [Internet]. 2021 [Citado 08 Abr 2021]; 17(1). Disponible en: http://ciberindex.com/index.php/p/article/view/e13091

15. Ramírez-Pérez $M$, Cárdenas-Jiménez $M$, Rodríguez-Jiménez $S$, Ramírez-Pérez $M$, Cárdenas-Jiménez M, Rodríguez-Jiménez S. El Dasein de los cuidados desde la fenomenología hermenéutica de Martín Heidegger. Enfermería Univ [Internet]. 2015 [Citado 08 Abr 2021]; 12(3): 144-51. Disponible en: https://dx.doi.org/10.1016/j.reu.2015.07.003

16. Dandicourt C. El cuidado de enfermería con enfoque en la comunidad. Rev Cuba Med Gen Integr [Internet]. 2018 [Citado 07 Abr 2021]; 34(1): 55-62. Disponible en: http://scielo.sld.cu/scielo.php?pid=S0864-21252018000100007\&script=sci_arttext\&tlng=pt

17. Shamsaei $F$, Yaghmaei $S$, Haghighi M. Exploring the lived experiences of the suicide attempt survivors: a phenomenological approach. Int ] Qual Stud Health Well-being [Internet]. 2020 [Citado 20 May 2021];15(1):1745478. Disponible en: https://pubmed.ncbi.nlm.nih.gov/32223374/

18. Perez-Toríz JE, Báez-Hernández J, Flores-Merlo M, Nava-Navarro V, Morales-Nieto A, ZentenoLópez Á. El significado del cuidado enfermero en el contexto de la pandemia COVID-19. SANUS [Internet]. 2020 [Citado 20 May 2021]; (16): 1-13. Disponible en: https://sanus.unison.mx/index.php/Sanus/article/view/236

19. Contreras-De-la-fuente HS, Castillo-Arcos LDC. Fenomenología: Una visión investigativa para enfermería. Cult los Cuid [Internet]. 2016 [Citado 20 May 2021]; 20(44): 15-24. Disponible en: https://doi.org/10.14198/cuid.2016.44.02

\section{Editor}

Escuela de Enfermería de la Universidad Católica Santo Toribio de Mogrovejo, Chiclayo, Perú

\section{Cómo citar este trabajo}

González-Soto C, Molina-Avilez D, Sabogal-Camargo F, Baca-Guido D. Aportes de la fenomenología interpretativa a las investigaciones de enfermería y al cuidado enfermero asistencial. Acc Cietna: para el cuidado de la salud [Internet]. 2021; 8(2): $95-105$. Disponible en: https://doi.org/10.35383/cietna.v8i2.664 


\section{Financiación}

El presente artículo no cuenta con financiación específica de agencias de financiamiento en los sectores público o privado para su desarrollo y/o publicación.

\section{Conflicto de interés}

Los autores del artículo declaran no tener ningún conflicto de intereses en su realización.

(C) Los autores. Este artículo es publicado por la Revista Acc Cietna: para el cuidado de la salud de la Escuela de Enfermería, Universidad Católica Santo Toribio de Mogrovejo.

Este es un artículo de acceso abierto, distribuido bajo los términos de la Licencia Creative Commons Atribución-NoComercial-CompartirIgual 4.0 Internacional (CC BY-NC-SA 4.0), que permite el uso no comercial, distribución y reproducción en cualquier medio, siempre que la obra original sea debidamente citada. 